\title{
Anomalous scaling of superrough growing surfaces: From correlation functions to residual local interfacial widths and scaling exponents
}

\author{
Ning-Ning Pang \\ Department of Physics, National Taiwan University, Taipei, Taiwan, Republic of China \\ Wen-Jer Tzeng \\ Department of Physics, Tamkang University, Tamsui, Taipei, Taiwan, Republic of China
}

(Received 5 February 2004; revised manuscript received 2 June 2004; published 24 September 2004)

\begin{abstract}
A study on the $(1+1)$-dimensional superrough growth processes is undertaken. We first work out the exact relations among the local interfacial width $w$, the correlation function $G$, and the $p$ th degree residual local interfacial width $w_{p}$ with $p=1,2,3, \ldots$. The relations obtained are exact and thus can be applied to any (1 +1 )-dimensional growth processes in the continuum limit, no matter whether the interface is superrough or not. Then we investigate the influence of the macroscopic structure formation on the scaling behavior of the superrough growth processes. Moreover, we show analytically that the residual local interfacial width $w_{p}$ excludes only the influence of the macroscopic structure on the scaling behavior of the system and retains the true scaling behavior originating from the stochastic nature of the system. Finally, we analyze and simulate some superrough growth models for demonstration.
\end{abstract}

DOI: 10.1103/PhysRevE.70.036115

PACS number(s): 02.50.Ey, 05.40.-a, 68.35.Ct, 81.10.Aj

\section{INTRODUCTION}

Kinetic interfacial roughening phenomena have been of great interest for the past two decades [1-4]. Recently, much attention has been focused on superrough growth processes because of their peculiar interfacial morphology [5-16]: the saturated global interfacial widths diverge faster than the system size $L$. The most distinct feature of the superrough interface is that its local scaling deviates from the usually Family-Vicsek dynamic scaling function [17]. This anomalous scaling behavior has been so widely observed numerically, analytically, and experimentally, ranging from molecular-beam epitaxial growth [5-8], interface advance in porous media [9], wood fracture surfaces [10], electrochemical deposition [11,12], even to brain tumor growth [13]. Among all the experimentally accessible quantities, one of the most informative quantities is the correlation function $G(r, t)$ defined as $\left\langle[h(x, t)-h(x+r, t)]^{2}\right\rangle_{L}$ with $h(x, t)$ denoting the interface height and $\langle\cdots\rangle_{L}$, throughout the paper, denoting the spatial average over the whole system of lateral size $L$ (there is also an implicit ensemble average when this is needed). Various superrough growth processes obey the following anomalous dynamic scaling ansatz [14]:

$$
G(r, t)=|r|^{2 \alpha} f\left(|r| / t^{1 / z},|r| / L\right)
$$

with the scaling function

$$
f\left(|r| / t^{1 / z},|r| / L\right) \sim\left\{\begin{array}{cc}
\left(|r| / t^{1 / z}\right)^{-2 \alpha} & \text { for }|r| \gg t^{1 / z} \\
\left(|r| / t^{1 / z}\right)^{-2 \kappa} & \text { for } L \gg t^{1 / z} \gg|r|, \\
(|r| / L)^{-2 \kappa} & \text { for } t^{1 / z} \gg L .
\end{array}\right.
$$

Here, the two independent scaling exponents $\alpha$ and $z$ are given the names roughness exponent and dynamic exponent, respectively. Note that the appearance of a third nonzero independent exponent $\kappa$ is the signature of this anomalous dy- namic scaling ansatz [14], distinct from the usual FamilyVicsek dynamic scaling ansatz [17].

The interface configurations of these superrough growth processes gradually develop global mountains or valleys (for example, see the figures in Refs. $[11,12,15]$ ), which have great influence on the scaling behavior of the system. This phenomenon can be easily understood: all these superrough growth processes are associated with local interfacial orientational instability but, at the same time, with fixed or periodic boundary conditions restricting the development of global interface tilt. Interestingly, various fluctuating time series problems such as physiological signals, atmospheric variability, currency exchange rates, and DNA sequences all show trends in addition to the statistical heterogeneity [18]. The generalized detrended fluctuation analysis method, proposed to systematically exclude the effect of trends by a power series expansion, has recently been numerically studied in detail in Ref. [18]. Expressed in terms of the Legendre polynomial, we analytically worked out the explicit expression for the trends of fluctuating systems to any order and pointed out the possibility of applying the generalized detrended fluctuation analysis method to superrough interfacial roughening processes in Ref. [19]. However, there still remain some unresolved issues. (1) The spirit of the generalized detrended fluctuation analysis method is to eliminate the influence of the background on the scaling behavior of the system. However, a rigorous proof is still lacking that this detrending operation itself will not alter the true scaling behavior originating from the stochastic nature of the system. (2) The explicit relations among the correlation function, the original local interfacial width, and the residual local interfacial width (relative to the macroscopic structure) have not been worked out. In this paper, we would like to work out the above two issues in detail. We will focus on $(1+1)$-dimensional superrough growth processes and explore the subtleties in extracting the scaling exponents. The outline 
of this paper is as follows. First, the exact relations among the local interfacial width $w$, the correlation function $G$, and the $p$ th degree residual local interfacial width $w_{p}$, with $p$ $=1,2,3, \ldots$, will be derived. Subsequently, we will explore their scaling relations and the influence of the macroscopic structure formation on the scaling exponents. Finally, the implications of the obtained results and their applicability to various fluctuating systems will be discussed.

\section{RESIDUAL LOCAL INTERFACIAL WIDTH AND CORRELATION FUNCTION}

First, let $h(x, t)$ denote the 1+1-dimensional interface height at a certain time $t$ and the lateral position $x$ in a continuous system. One realization of the local interfacial width in an observation window, centered at $\hat{x}$, of length $l(\ll L)$ is then given by

$$
w^{2}(l ; \hat{x} ; t) \equiv\left\langle\left[h(x, t)-\langle h(x, t)\rangle_{l ; \hat{x}}\right]^{2}\right\rangle_{l ; \hat{x}}
$$

with $\langle\cdots\rangle_{l ; \hat{x}}$ denoting throughout this paper the spatial average over an observation window, centered at $\hat{x}$, of length $l$. Using a $p$ th degree polynomial to extract the contribution from macroscopic structure formation, the corresponding $p$ th degree residual local interfacial width in that observation window of length $l$ is defined as

$$
w_{p}^{2}(l ; \hat{x} ; t) \equiv\left\langle\left[h(x, t)-\hat{h}_{p}(x ; \hat{x} ; t)\right]^{2}\right\rangle_{l ; \hat{x}}
$$

where the $p$ th degree polynomial $\hat{h}_{p}(x ; \hat{x} ; t)$ is obtained from a least squares fit to $h(x, t)$ within that observation window. Figure 1 gives a pictorial illustration about the above related quantities. Since the Legendre polynomial $P_{q}(x)$ is a polynomial of degree $q$ and satisfies the orthogonal relations [20]

$$
\int_{-1}^{1} d x P_{q}(x) P_{q^{\prime}}(x)=\frac{2}{2 q+1} \delta_{q, q^{\prime}},
$$

we thus choose the Legendre polynomial $P_{q}(2(x-\hat{x}) / l)$ to be the basis of $\hat{h}_{p}(x ; \hat{x} ; t)$; namely,

$$
\hat{h}_{p}(x ; \hat{x} ; t)=\sum_{q=0}^{p} C_{q} P_{q}\left(\frac{2(x-\hat{x})}{l}\right)
$$

with the coefficient

$$
C_{q}=(2 q+1)\left\langle h(x, t) P_{q}\left(\frac{2(x-\hat{x})}{l}\right)\right\rangle_{l ; \hat{x}},
$$

obtained from the relation

$$
\frac{\partial w_{p}^{2}(l ; \hat{x} ; t)}{\partial C_{q}}=0 .
$$

To be self-contained, we listed Eqs. (4)-(7), which were derived in Ref. [19]. For details of the derivation, see Ref. [19].

Now we will employ the above results to work out explicit and exact relations among the original local interfacial width, the correlation function, and the $p$ th degree residual local interfacial width. From the definition of $G(r, t)$ and

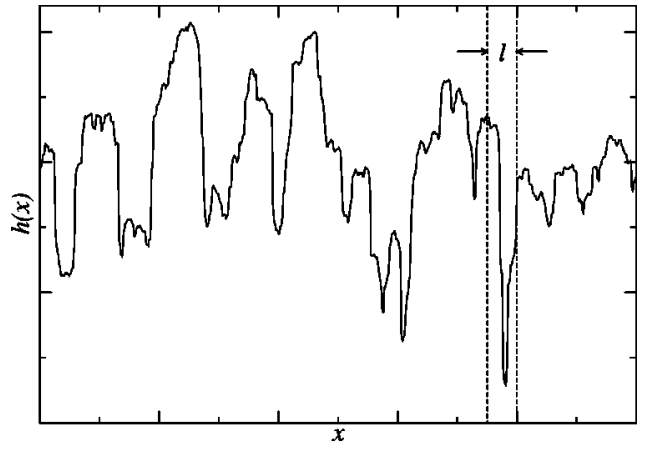

(a)

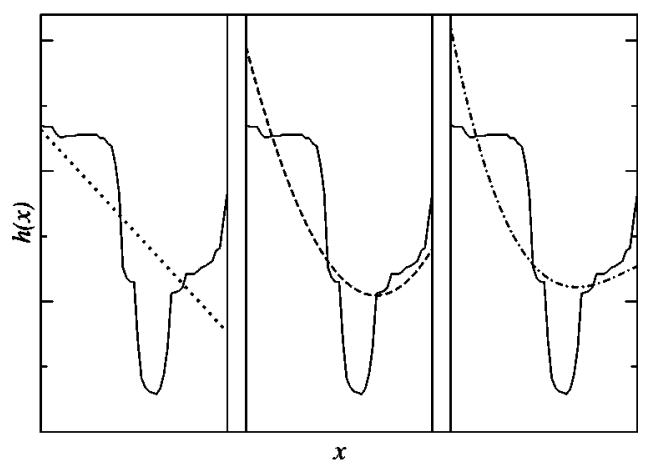

(b)

FIG. 1. (a) A typical interface configuration of $(1+1)$-dimensional superrough growth processes. (b) The solid curve represents the interfacial configuration $h(x)$ within a local window of size $l$ ( $\ll$ the lateral system size $L$ ) extracted from (a). The dotted, dashed, and dash-dotted curves in the left, middle, and right sections represent the $p$ th degree polynomial $\hat{h}_{p}(x)$ with $p=1$, 2 , and 3 , respectively, obtained from least squares fits to the interfacial configuration in that local window.

$\left\langle w^{2}(l ; \hat{x} ; t)\right\rangle_{L}$, it is straightforward to obtain the relation [16]

$$
\left\langle w^{2}(l ; \hat{x} ; t)\right\rangle_{L}=\frac{1}{l^{2}} \int_{0}^{l} d r(l-r) G(r, t) .
$$

Subsequently, by applying Eqs. (6) and (7), it is straightforward to derive the relation $\left\langle\left[h(x, t)-\hat{h}_{p}(x ; \hat{x} ; t)\right] \hat{h}_{p}(x ; \hat{x} ; t)\right\rangle_{l ; \hat{x}}$ $=0$. Thus,

$$
\begin{aligned}
w_{p}^{2}(l ; \hat{x} ; t) & \equiv\left\langle\left[h(x, t)-\hat{h}_{p}(x ; \hat{x} ; t)\right]^{2}\right\rangle_{l ; \hat{x}} \\
& =\left\langle\left[h(x, t)-\hat{h}_{p}(x ; \hat{x} ; t)\right] h(x, t)\right\rangle_{l ; \hat{x}} \\
& =\left\langle h^{2}(x, t)\right\rangle_{l ; \hat{x}}-\sum_{q=0}^{p} \frac{1}{2 q+1} C_{q}^{2} .
\end{aligned}
$$

By using the definition of $w^{2}(l ; \hat{x} ; t)$ and Eq. (7), we then obtain the relation between $w^{2}(l ; \hat{x} ; t)$ and $w_{p}^{2}(l ; \hat{x} ; t)$ as follows: 


$$
w_{p}^{2}(l ; \hat{x} ; t)=w^{2}(l ; \hat{x} ; t)-\sum_{q=1}^{p} \frac{1}{2 q+1} C_{q}^{2} .
$$

Then we will employ the above obtained results to derive the exact relation between $\left\langle w_{p}^{2}(l ; \hat{x} ; t)\right\rangle_{L}$ and $G(r, t)$. From Eq. (7),

$$
\begin{aligned}
\left\langle C_{q \geqslant 1}^{2}\right\rangle_{L}= & \frac{(2 q+1)^{2}}{l^{2}} \int_{-l / 2}^{l / 2} d r^{\prime} \int_{-l / 2}^{l / 2} d r^{\prime \prime} P_{q}\left(\frac{2 r^{\prime}}{l}\right) P_{q}\left(\frac{2 r^{\prime \prime}}{l}\right)\langle h(x \\
& \left.\left.+r^{\prime}, t\right) h\left(x+r^{\prime \prime}, t\right)\right\rangle_{L} .
\end{aligned}
$$

By employing the definition of $G\left(r^{\prime}-r^{\prime \prime}, t\right)$ and the wellknown property of $P_{q}(x)$ [20] $\int_{-1}^{1} P_{q}(x) d x=0$ for all $q \geqslant 1$, we then have

$$
\begin{aligned}
\left\langle C_{q \geqslant 1}^{2}\right\rangle_{L}= & -\frac{(2 q+1)^{2}}{2 l^{2}} \int_{-l / 2}^{l / 2} d r^{\prime} \int_{-l / 2}^{l / 2} d r^{\prime \prime} P_{q}\left(\frac{2 r^{\prime}}{l}\right) P_{q}\left(\frac{2 r^{\prime \prime}}{l}\right) \\
& \times G\left(r^{\prime}-r^{\prime \prime}, t\right) \\
= & -\frac{(2 q+1)^{2}}{l^{2}} \int_{0}^{l} d r G(r, t) \int_{-l / 2}^{l / 2-r} d r^{\prime \prime} P_{q}\left(\frac{2\left(r+r^{\prime \prime}\right)}{l}\right) \\
& \times P_{q}\left(\frac{2 r^{\prime \prime}}{l}\right) .
\end{aligned}
$$

Substituting Eqs. (9) and (13) into Eq. (11), we consequently obtain the exact relation between $\left\langle w_{p}^{2}(l ; \hat{x} ; t)\right\rangle_{L}$ and $G(r, t)$ as

$$
\left\langle w_{p}^{2}(l ; \hat{x} ; t)\right\rangle_{L}=\frac{1}{l^{2}} \int_{0}^{l} d r G(r, t) K_{p}(r)
$$

with the kernel

$$
K_{p}(r)=\sum_{q=0}^{p}(2 q+1) \int_{-l / 2}^{l / 2-r} d r^{\prime \prime} P_{q}\left(\frac{2\left(r+r^{\prime \prime}\right)}{l}\right) P_{q}\left[\frac{2 r^{\prime \prime}}{l}\right] .
$$

Thus, we have succeeded in obtaining the exact relations among $\left\langle w^{2}(l ; \hat{x} ; t)\right\rangle_{L}, G(r, t)$, and $\left\langle w_{p}^{2}(l ; \hat{x} ; t)\right\rangle_{L}$. The relations obtained are exact and thus can be applied to any $(1+1)$-dimensional growth processes in the continuum limit, no matter whether the interface is superrough or not.

We will further explore the properties of the kernel $K_{p}(r)$, since they have a great influence on the scaling relations among $\left\langle w^{2}(l ; \hat{x} ; t)\right\rangle_{L}, G(r, t)$, and $\left\langle w_{p}^{2}(l ; \hat{x} ; t)\right\rangle_{L}$. The kernel $K_{p}(r)$ can be recast as follows:

$$
K_{p}(r)=\sum_{q=0}^{p}\left[l-(2 q+1) \int_{0}^{r} d \hat{r} P_{q}\left(1-2\left(\frac{\hat{r}}{l}\right)^{2}\right)\right] .
$$

The detailed derivation of Eq. (16) from Eq. (15) is given in Appendix A. Then, from Eq. (16) and the definition of Legendre polynomial $P_{q}(x)$, it is straightforward to tell that the kernel $K_{p}(r)$ is a polynomial of $r$ with terms of odd power up to degree $2 p+1$ plus a constant term. For illustration, $K_{0}(r)$ to $K_{3}(r)$ are explicitly listed as follows:

$$
\begin{gathered}
K_{0}(r)=l-r, \\
K_{1}(r)=2 l-4 r+2 r^{3} / l^{2}, \\
K_{2}(r)=3 l-9 r+12 r^{3} / l^{2}-6 r^{5} / l^{4}, \\
K_{3}(r)=4 l-16 r+40 r^{3} / l^{2}-48 r^{5} / l^{4}+20 r^{7} / l^{6} .
\end{gathered}
$$

The most important feature of the kernel $K_{p}(r)$ is as follows: with $\beta$ an arbitrary positive number (including nonintegers),

$$
\int_{0}^{l} d r r^{2 \beta} K_{p}(r)=\left\{\begin{array}{cc}
0 & \text { for } \beta=1,2, \ldots, p \\
l^{2 \beta+2}\left[\frac{(-1)^{p}(p+1) \Gamma(\beta) \Gamma(\beta+1)}{(4 \beta+2) \Gamma(\beta-p) \Gamma(\beta+p+2)}\right] & \text { otherwise. }
\end{array}\right.
$$

The detailed derivation is given in Appendix B.

Now, we will apply the above obtained results to investigate the scaling relations among $\left\langle w^{2}(l ; \hat{x} ; t)\right\rangle_{L}, G(r, t)$, and $\left\langle w_{p}^{2}(l ; \hat{x} ; t)\right\rangle_{L}$ in detail. We first rewrite $h(x, t)$ as

$$
h(x, t)=h_{\text {macro }}(x, t)+h_{\text {sto }}(x, t) \text {, }
$$

in which $h_{\text {macro }}(x, t)$ denotes the height of the macroscopic structure at position $x$ and time $t$, and $h_{\text {sto }}(x, t)$ denotes the noise-induced height fluctuation relative to the macroscopic structure. By substituting the above expression for $h(x, t)$ into the definition of $G(r, t)$, it is straightforward to obtain

$$
G(r, t)=G_{\text {macro }}(r, t)+G_{\text {sto }}(r, t)
$$

with $G_{\text {macro }}(r, t)$ and $G_{\text {sto }}(r, t)$ denoting the contributions from the macroscopic structure and from the noise-induced fluctuation relative to the macroscopic structure, respectively. Supposing the macroscopic structure to be continuous and smooth, the term $G_{\text {macro }}(r, t)$ is expected to be analytic in $r$. Note that $G(r, t)$ is a difference correlation function, so one has $G(-r, t)=G(r, t)$ and $G(0, t)=0$. Thus, the power series expansion of $G_{\text {macro }}(r, t)$ should contain only those terms with even power without the constant term, i.e., $G_{\text {macro }}(r, t)$ $=\sum_{q=1}^{\infty} \hat{A}_{2 q}(t) r^{2 q}$. In addition, suppose that $G_{\text {sto }}(r, t)$ is equal to $\hat{A}_{2 \alpha}(t)|r|^{2 \alpha}$, with $\alpha$ being a positive noninteger number, dis- 
tinct from the macroscopic structure. From Eq. (9), we have

$$
\begin{aligned}
\left\langle w^{2}(l ; \hat{x} ; t)\right\rangle_{L}= & \frac{1}{l^{2}} \int_{0}^{l} d r(l-r)\left[G_{\text {macro }}(r, t)+G_{\text {sto }}(r, t)\right] \\
= & \sum_{q=1}^{\infty}\left[\frac{\hat{A}_{2 q}(t)}{(2 q+1)(2 q+2)}\right] l^{2 q} \\
& +\left[\frac{\hat{A}_{2 \alpha}(t)}{(2 \alpha+1)(2 \alpha+2)}\right] l^{2 \alpha} .
\end{aligned}
$$

We see that the scaling behavior of $\left\langle w^{2}(l ; \hat{x} ; t)\right\rangle_{L}$ is exactly the same as that of $G(r, t)$. In contrast, by employing Eq. (14) and the above obtained most important feature of $K_{p}(r)$ given in Eq. (18), we have

$$
\begin{aligned}
\left\langle w_{p}^{2}(l ; \hat{x} ; t)\right\rangle_{L}= & \frac{1}{l^{2}} \int_{0}^{l} d r K_{p}(r)\left[G_{\text {macro }}(r, t)+G_{\text {sto }}(r, t)\right] \\
= & \sum_{q=p+1}^{\infty}\left[\frac{(-1)^{p}(p+1) \Gamma(q) \Gamma(q+1) \hat{A}_{2 q}(t)}{(4 q+2) \Gamma(q-p) \Gamma(q+p+2)}\right] l^{2 q} \\
& +\left[\frac{(-1)^{p}(p+1) \Gamma(\alpha) \Gamma(\alpha+1) \hat{A}_{2 \alpha}(t)}{(4 \alpha+2) \Gamma(\alpha-p) \Gamma(\alpha+p+2)}\right] l^{2 \alpha} .
\end{aligned}
$$

Indeed, the terms $\left(l^{2}, l^{4}, \ldots, l^{2 p}\right)$ in $\left\langle w^{2}(l ; \hat{x} ; t)\right\rangle_{L}$ due to macroscopic structure formation have been successfully suppressed here and, at the same time, the term $l^{2 \alpha}$ due to the stochastic nature still remains. Consequently, we have rigorously shown that the residual local interfacial width $\left\langle w_{p}^{2}(l ; \hat{x} ; t)\right\rangle_{L}$ excludes only the influence of the macroscopic structure on the scaling behavior of the system and retains the true scaling behavior originating from the stochastic nature of the system.

\section{APPLICATIONS}

In the following, we will apply the above obtained relations to some interfacial growth processes. First, we consider the following interfacial growth equations in $1+1$ dimensions [6]:

$$
\partial_{t} h(x, t)=(-1)^{m+1} \nu \partial_{x}^{2 m} h(x, t)+\eta(x, t)
$$

with integer $m \geqslant 2$, where $\eta(x, t)$ denotes white noise with zero mean. By using a scaling analysis, it is straightforward to obtain the dynamic exponent $z=2 \mathrm{~m}$ and the roughness exponent $\alpha=(2 m-1) / 2$. For integer $m \geqslant 2$, the roughness exponent $\alpha>1$ and, thus, the interfacial growth processes described by the above class of growth equations display superroughening phenomena. The asymptotes of the correlation function $G(r, t)$ in the intermediate time regime $|r|^{z} / \nu \ll t \ll L^{z} / \nu$ and the late time regime $t \gg L^{z} / \nu$ are given by

$$
\left.G(r, t)\right|_{|r| z / \nu \ll t \ll L / \nu} \simeq \sum_{q=1}^{\infty} A_{2 q}^{\prime} t^{2(\alpha-q) / z} r^{2 q}+A_{2 \alpha}^{\prime}|r|^{2 \alpha}
$$

and

$$
\left.G(r, t)\right|_{t \gg L^{z / \nu}} \simeq \sum_{q=1}^{m} B_{2 q}^{\prime} L^{2(\alpha-q)} r^{2 q}+B_{2 \alpha}^{\prime}|r|^{2 \alpha},
$$

respectively, with $L$ denoting the lateral system size. The detailed derivation was given in Ref. [6]. Note that the terms $\left(t^{2(\alpha-1) / z} r^{2}, t^{2(\alpha-2) / z} r^{4}, \ldots, t^{2(\alpha-m+1) / z} r^{2 m-2}\right) \quad$ and $\left(L^{2(\alpha-1)} r^{2}, L^{2(\alpha-2)} r^{4}, \ldots, L^{2(\alpha-m+1)} r^{2 m-2}\right)$ due to the macroscopic structure formation in the intermediate time regime $|r|^{z} / \nu \ll t \ll L^{z} / \nu$ and the late time regime $t \gg L^{z} / \nu$, respectively, are all dominant over the true scaling term $|r|^{2 \alpha}$ originating from the stochastic nature of the system. As we showed in the previous section, the square of the local interfacial width $\left\langle w^{2}(l ; \hat{x} ; t)\right\rangle_{L}$ will have exactly the same asymptotic behavior as that of the correlation function $G(r, t)$, namely,

$$
\left.\left\langle w^{2}(l ; \hat{x} ; t)\right\rangle_{L}\right|_{l z / \nu \ll t \ll L / \nu} \simeq \sum_{q=1}^{\infty} A_{2 q}^{\prime \prime} t^{2(\alpha-q) / z} l^{2 q}+A_{2 \alpha}^{\prime \prime} l^{2 \alpha}
$$

and

$$
\left.\left\langle w^{2}(l ; \hat{x} ; t)\right\rangle_{L}\right|_{t \gg L^{z / \nu}} \simeq \sum_{q=1}^{m} B_{2 q}^{\prime \prime} L^{2(\alpha-q)} l^{2 q}+B_{2 \alpha}^{\prime \prime} l^{2 \alpha} .
$$

Thus, the terms $\left(t^{2(\alpha-1) / z} l^{2}, t^{2(\alpha-2) / z} l^{4}, \ldots, t^{2(\alpha-m+1) / z} l^{2 m-2}\right)$ and $\left(L^{2(\alpha-1)} l^{2}, L^{2(\alpha-2)} l^{4}, \ldots, L^{2(\alpha-m+1)} l^{2 m-2}\right)$ due to the macroscopic structure formation in the intermediate time regime $l^{z} / \nu \ll t \ll L^{z} / \nu$ and the late time regime $t \gg L^{z} / \nu$, respectively, are all dominant over the true scaling term $l^{2 \alpha}$.

Since the leading anomalous terms in the intermediate and late time asymptotes of the square of the local interfacial width are simply polynomials of $l$ with even power from $l^{2}$ up to $l^{2 m-2}$, the $(m-1)$ th order detrending of the original interface configuration [i.e., the subtraction of $\hat{h}_{m-1}(x, t)$ $=\sum_{q=0}^{m-1} C_{q}(t) P_{q}(2(x-\hat{x}) / l)$ from $\left.h(x, t)\right]$ is necessary in order to extract the roughness exponent from the true scaling term originating from the stochastic nature of the system. For systems obeying conventional Family-Vicsek scaling [17], several works [21-24] in the literature have pointed out that the typical method of measuring the roughness exponents, i.e., $\quad \log _{10}\left[G(r, t) / G\left(r^{\prime}, t\right)\right] /\left.2 \log _{10}\left(|r| /\left|r^{\prime}\right|\right)\right|_{t \gg|r|^{z} \text { and }\left|r^{\prime}\right|^{z}}$

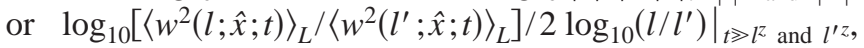
does not give reliable estimates in practical situations with finite time, finite system size, and finite spatial resolution. In contrast, for systems with macroscopic structure formation, the typical method does not give correct estimates of the roughness exponent even in the continuum limit (i.e., large system size and large time). For systems obeying conventional Family-Vicsek scaling, the detrending operation cannot improve the deviation of the numerically estimated scaling exponents from the theoretical values (due to finite size and/or finite time effects), while for systems with macroscopic structure formation, the detrending operation is a "must do" in order to get reliable estimates of scaling exponents.

Next, we study the curvature model [24] in $1+1$ dimensions. The growth rule is given as follows. (1) A site is cho- 


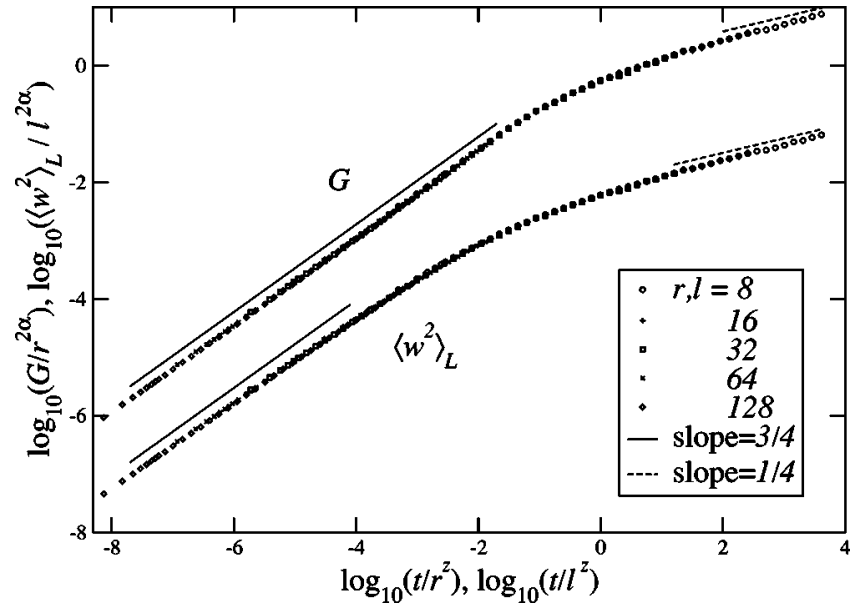

FIG. 2. The $\log -\log$ plots of $G(r, t) / r^{2 \alpha}$ vs $t / r^{z}$ and $\left\langle w^{2}(l ; \hat{x} ; t)\right\rangle_{L} / l^{2 \alpha}$ vs $t / l^{z}$ with $\alpha=3 / 2$ and $z=4$ for the $(1+1)$-dimensional curvature model. The solid and dashed lines with the slopes $3 / 4$ and $1 / 4$, respectively, are merely drawn for a guide to the eye.

sen randomly. (2) The freshly landed atom relaxes immediately to the site with the largest local curvature $h_{i-1}+h_{i+1}$ $-2 h_{i}$ among this site and its nearest neighbors. We first measure the local interfacial width and the equal-time height difference correlation function with the system size $L=8192$ sites and averaged over 20 realizations. In Fig. 2, we show the excellent data collapse of the scaled $G(r, t) / r^{2 \alpha}$ vs $t / r^{z}$ and $\left\langle w^{2}(l ; \hat{x} ; t)\right\rangle_{L} / l^{2 \alpha}$ vs $t / l^{z}$ with the roughness exponent $\alpha$ $=3 / 2$ and the dynamic exponent $z=4$. The data for the local interfacial width are shifted downward by one unit for visibility. The nonsaturation of the scaling functions in Fig. 2 indicates that this system obeys the anomalous dynamic scaling ansatz described by Eqs. (1) and (2). Since the interface of the curvature model gradually develops a macroscopic structure, we then numerically measure the $p$ th degree residual local interfacial width with $p=1$ or 2 for the same system size and realization number as those in Fig. 2. Figure 3 is a log-log plot of the scaled $\left\langle w_{p}^{2}(l ; \hat{x} ; t)\right\rangle_{L} / l^{2 \alpha}$ vs $t / l^{z}$ with $p=1$ or 2 , where the data for $p=2$ are shifted downward by one unit for visibility. Note that, in the continuum limit, the first order detrending of the curvature model should be sufficient to suppress the leading anomalous term and let the residual local interfacial width retrieve the conventional Family-Vicsek scaling, i.e., reach saturation when $t \gg l^{2}$. However, for finite size and finite time, $\left\langle w_{1}^{2}(l ; \hat{x} ; t)\right\rangle_{L}$ suffers from a long crossover before retrieving the conventional scaling; in contrast, the transition of $\left\langle w_{2}^{2}(l ; \hat{x} ; t)\right\rangle_{L}$ from the transient regime to the saturated regime is much sharper, as can be seen in Fig. 3 .

Hence, to deal with systems with finite time and finite size (as in experiments), a higher order (than the theoretical prediction) detrending operation is helpful for removing the influence of macroscopic structure formation on the scaling behaviors of superrough interfaces. To demonstrate this point more explicitly, we measure the quantity

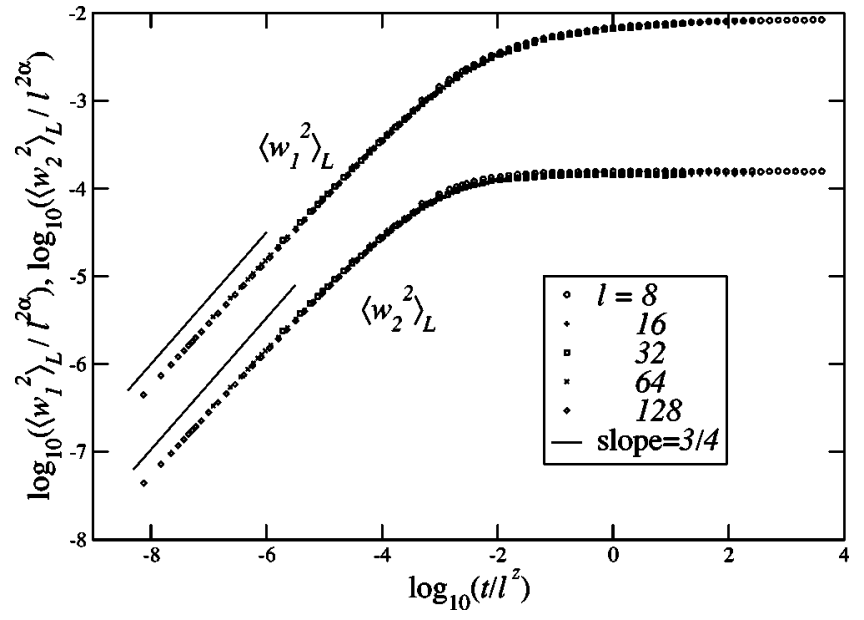

FIG. 3. The log-log plots of $\left\langle w_{1}^{2}(l ; \hat{x} ; t)\right\rangle_{L} / l^{2 \alpha}$ and $\left\langle w_{2}^{2}(l ; \hat{x} ; t)\right\rangle_{L} / l^{2 \alpha}$ vs $t / l^{z}$ with $\alpha=3 / 2$ and $z=4$ for the $(1+1)$-dimensional curvature model.

$$
\alpha_{l_{1} / l_{2}, p}(t) \equiv \log _{10}\left(\frac{\left\langle w_{p}^{2}\left(l_{1} ; \hat{x} ; t\right)\right\rangle_{L}}{\left\langle w_{p}^{2}\left(l_{2} ; \hat{x} ; t\right)\right\rangle_{L}}\right) / 2 \log _{10}\left(\frac{l_{1}}{l_{2}}\right)
$$

with $p=1$ or 2 . Figure 4 shows the effective roughness exponent $\alpha_{l_{1} / l_{2}, p}(t)$ vs the rescaled time $t / l_{1}^{z}$ with $z=4$ in a semilogarithmic plot. Note that the result for $p=2$ is shifted upward by 0.5 units for visibility. The simulation is done with a lateral system size $L=8192$ and averaged over 12 runs. The results indicate that the value of $\alpha_{l_{1} / l_{2, p=1}}$ is largely influenced by the finite time effect and has not reached saturation even at $t>10^{3} l_{1}^{z}$, which is 4096000 even for a quite small value of $l_{1}=8$. In contrast, the value of $\alpha_{l_{1} l_{2, p=2}}$ reaches saturation at $t \sim l_{1}^{z}$ as expected and the finite time effect is negligible. For completeness, we also study the finite size effect. Figure 5 shows the effective roughness exponent $\alpha_{8 / 16, p=2}$ vs time $t$ with several different values of $L$ in a semilogarithmic plot. The simulation is done with realizations equal to 60,20 , and

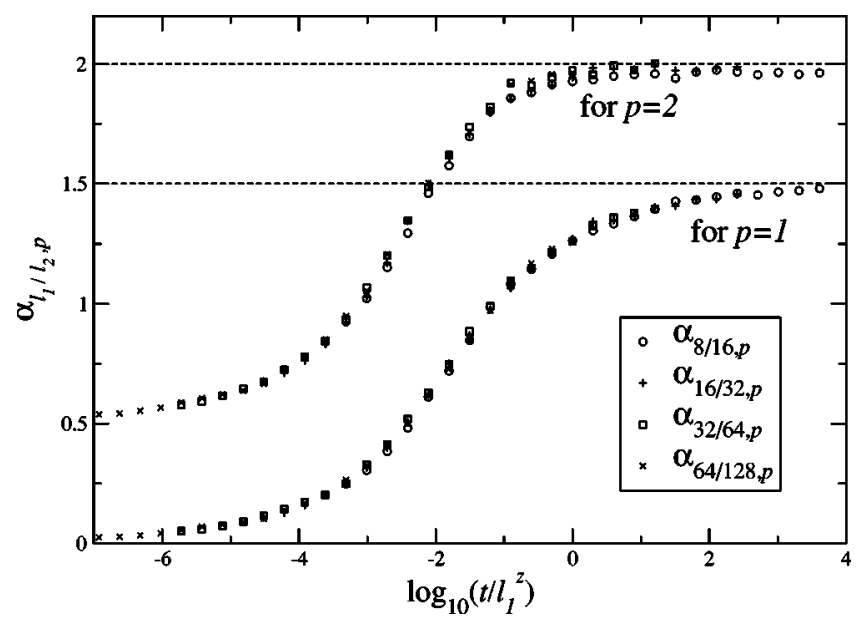

FIG. 4. The effective roughness exponent $\alpha_{l_{1} / l_{2, p}}(t)$ vs the rescaled time $t / l_{1}^{z}$ with $z=4$ in a semilogarithmic plot for the $(1+1)$-dimensional curvature model. Note that the result for $p=2$ is shifted upward by 0.5 units for visibility. 


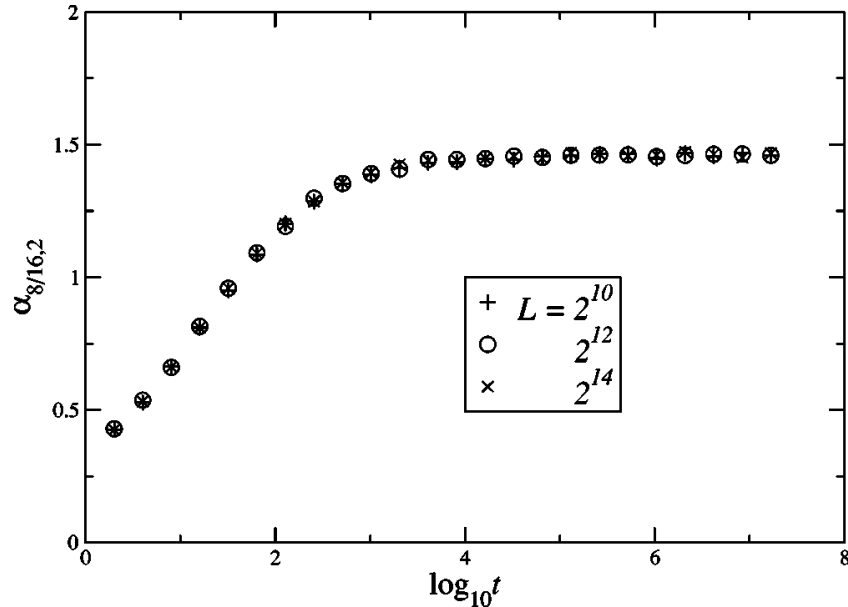

FIG. 5. The effective roughness exponent $\alpha_{8 / 16,2}(t)$ vs time $t$ with several different lateral system sizes $L$ in a semilogarithmic plot for the $(1+1)$-dimensional curvature model.

5 for the lateral system size $L=2^{10}, 2^{12}$, and $2^{14}$, respectively. The results indicate that the finite size effect does not have a significant influence on the values of the effective roughness exponents $\alpha_{l_{1} / l_{2}, p}$, as can be seen in Fig. 5. In other words, the residual local interfacial width $w_{p}$ is used to extract the true scaling originating from the stochastic nature of the system by discarding the influence of macroscopic structure. When measuring the scaling exponents of superrough interfaces in experiments, the first, second, etc., order detrending procedures should be performed in turn until the results are stable, i.e., independent of the finite time effect.

\section{CONCLUSION}

In conclusion, we undertook a study on $(1+1)$-dimensional superrough growth processes. We first worked out the exact relations among the local interfacial width $w$, the correlation function $G$, and the $p$ th degree residual local interfacial width $w_{p}$ with $p=1,2,3, \ldots$. The relations obtained are exact and thus can be applied to any $(1+1)$-dimensional growth processes in the continuum limit, no matter whether the interface is superrough or not. Then, an investigation of the influence of the macroscopic structure formation on the scaling behavior of the superrough growth processes is undertaken. In addition, we explicitly showed that the residual local interfacial width $w_{p}$ excludes only the influence of the macroscopic structure on the scaling behavior of the system and retains the true scaling behavior originating from the stochastic nature of the system. Finally, we studied a class of linear growth equations Eq. (23) and a superrough growth model (the curvature model) for demonstration.

\section{ACKNOWLEDGMENTS}

The work of N.-N.P. is supported in part by the National Science Council of the Republic of China under Grant No. NSC-92-2112-M002-017. The work of W.-J.T. is supported in part by the National Science Council of the Republic of China under Grant No. NSC-92-2112-M032-011.

\section{APPENDIX A}

Equation (15) can be first recast as follows:

$$
\begin{aligned}
K_{p}(r)= & \sum_{q=0}^{p}\left[(2 q+1) \int_{-l / 2}^{l / 2} d r^{\prime \prime} P_{q}\left(\frac{2\left(r+r^{\prime \prime}\right)}{l}\right) P_{q}\left(\frac{2 r^{\prime \prime}}{l}\right)\right. \\
& \left.-(2 q+1) \int_{l / 2-r}^{l / 2} d r^{\prime \prime} P_{q}\left(\frac{2\left(r+r^{\prime \prime}\right)}{l}\right) P_{q}\left(\frac{2 r^{\prime \prime}}{l}\right)\right] \\
\equiv & \sum_{q=0}^{p}\left[A_{q}-B_{q}\right] .
\end{aligned}
$$

From the definition of the Legendre polynomial $P_{q}(x)$ and the orthogonal relation [20]

$$
\int_{-1}^{1} x^{n} P_{q}(x) d x=\left\{\begin{array}{cc}
0 & \text { for } n=0,1, \ldots, q-1 \\
2\left(\frac{q !}{(2 q+1) ! !}\right) & \text { for } n=q
\end{array}\right.
$$

it is straightforward to obtain

$$
A_{q}=l \text {. }
$$

The derivation of $B_{q}$ is much more complicated. First, by a change of variable $\widetilde{r} \equiv 1 / 2-r^{\prime \prime} / l$, we have

$$
B_{q}=(2 q+1) l \int_{0}^{r / l} d \widetilde{r} P_{q}(1+2(r / l)-2 \widetilde{r}) P_{q}(1-2 \widetilde{r}) .
$$

Subsequently, by substituting the known relation [20]

$$
P_{q}(1+2 x)=\sum_{m=0}^{q}\left(\begin{array}{c}
q \\
m
\end{array}\right)\left(\begin{array}{c}
q+m \\
m
\end{array}\right) x^{m}
$$

into the above equation and with some tedious calculation, we obtain

$$
\begin{aligned}
B_{q}= & (2 q+1) l \sum_{n=0}^{2 q} \frac{(r / l)^{n+1}}{(n+1) !} \\
& \times\left[\sum_{m=0}^{n} \frac{(-1)^{m}(q+n-m) !(q+m) !}{(q-n+m) !(q-m) ! m !(n-m) !}\right] \\
= & (2 q+1) l \sum_{n=0}^{2 q} \frac{(r / l)^{n+1}}{(n+1) !} t_{n}(2 q+1, q),
\end{aligned}
$$

where $t_{n}$ is the Chebyshev polynomial [20]. By employing the property 


$$
\begin{gathered}
t_{2 n+1}(2 q+1, q)=0, \\
t_{2 n}(2 q+1, q)=(-1)^{n}(2 n) !\left(\begin{array}{c}
2 n \\
n
\end{array}\right)\left(\begin{array}{c}
q+n \\
2 n
\end{array}\right),
\end{gathered}
$$

we then obtain

$$
B_{q}=(2 q+1) l \sum_{n=0}^{q} \frac{(-1)^{n}}{2 n+1}\left(\begin{array}{c}
q \\
n
\end{array}\right)\left(\begin{array}{c}
q+n \\
n
\end{array}\right)\left(\frac{r}{l}\right)^{2 n+1} .
$$

Finally, by using the relation given in Eq. (A4), $B_{q}$ is then recast as

$$
B_{q}=(2 q+1) \int_{0}^{r} P_{q}\left(1-2\left(\frac{\hat{r}}{l}\right)^{2}\right) d \hat{r}
$$

Consequently, the kernel $K_{p}(r)$ is derived as Eq. (16).

\section{APPENDIX B}

Here, we will derive the most important feature of the kernel $K_{p}(r)$, Eq. (18). First, with $\beta$ being a positive number and substituting Eq. (16) for $K_{p}(r)$, we have

$$
\begin{aligned}
\int_{0}^{l} d r r^{2 \beta} K_{p}(r)= & \sum_{q=0}^{p}\left[\frac{l^{2 \beta+2}}{2 \beta+1}-(2 q+1)\right. \\
& \left.\times \int_{0}^{l} d \hat{r} \int_{\hat{r}}^{l} d r r^{2 \beta} P_{q}\left(1-2\left(\frac{\hat{r}}{l}\right)^{2}\right)\right] \\
= & \sum_{q=0}^{p}\left[\frac{l^{2 \beta+2}}{2 \beta+1}-\left(\frac{2 q+1}{2 \beta+1}\right) l^{2 \beta+1}\right. \\
& \times \int_{0}^{l} d \hat{r} P_{q}\left(1-2\left(\frac{\hat{r}}{l}\right)^{2}\right)+\left(\frac{2 q+1}{2 \beta+1}\right) \\
& \left.\times \int_{0}^{l} d \hat{r} \hat{r}^{2 \beta+1} P_{q}\left(1-2\left(\frac{\hat{r}}{l}\right)^{2}\right)\right] \\
\equiv & \sum_{q=0}^{p}\left[\tilde{A}_{q}+\tilde{B}_{q}+\tilde{C}_{q}\right] .
\end{aligned}
$$

With a change of variable $\widetilde{r} \equiv 1-2(\hat{r} / l)^{2}, \widetilde{A}_{q}, \widetilde{B}_{q}$, and $\widetilde{C}_{q}$ can be recast as follows:

$$
\begin{gathered}
\tilde{A}_{q}=\frac{l^{2 \beta+2}}{2 \beta+1}, \\
\widetilde{B}_{q}=-\left(\frac{l^{2 \beta+2}}{2 \beta+1}\right)\left(\frac{2 q+1}{2 \sqrt{2}}\right) \int_{-1}^{1} d \widetilde{r} \frac{P_{q}(\widetilde{r})}{\sqrt{1-\tilde{r}}} \\
\widetilde{C}_{q}=\left(\frac{l^{2 \beta+2}}{2 \beta+1}\right)\left(\frac{2 q+1}{4}\right) \int_{-1}^{1} d \widetilde{r}\left(\frac{1-\tilde{r}}{2}\right)^{\beta} P_{q}(\widetilde{r}) .
\end{gathered}
$$

Next, by employing the relation [20]

$$
\int_{-1}^{1} d \tilde{r} \frac{P_{q}(\tilde{r})}{\sqrt{1-\tilde{r}}}=\frac{2 \sqrt{2}}{2 q+1}
$$

we then have $\widetilde{A}_{q}=-\widetilde{B}_{q}=l^{2 \beta+2} /(2 \beta+1)$ and thus

$$
\begin{aligned}
\int_{0}^{l} d r r^{2 \beta} K_{p}(r)= & \sum_{q=0}^{p} \widetilde{C}_{q} \\
= & \frac{1}{4}\left(\frac{l^{2 \beta+2}}{2 \beta+1}\right) \int_{-1}^{1} d \widetilde{r}\left(\frac{1-\widetilde{r}}{2}\right)^{\beta} \\
& \times\left[\sum_{q=0}^{p}(2 q+1) P_{q}(\widetilde{r})\right] \\
= & \frac{l^{2 \beta+2}(p+1)}{2^{\beta+2}(2 \beta+1)} \int_{-1}^{1} d \widetilde{r}(1-\widetilde{r})^{\beta-1} \\
& \times\left[P_{p}(\widetilde{r})-P_{p+1}(\widetilde{r})\right]
\end{aligned}
$$

here we have used the relation [20]

$$
\sum_{q=0}^{p}(2 q+1) P_{q}(\widetilde{r})=\frac{(p+1)\left[P_{p}(\widetilde{r})-P_{p+1}(\widetilde{r})\right]}{1-\tilde{r}}
$$

in deriving the last expression in Eq. (B3).

(i) For $\beta=1,2, \ldots, p$, by employing the orthogonal relation for the Legendre polynomial given in Eq. (A2), we easily obtain $\int_{-1}^{1} d \widetilde{r}(1-\widetilde{r})^{\beta-1} P_{p(\text { or } p+1)}(\widetilde{r})=0$ and, thus, $\int_{0}^{l} d r r^{2 \beta} K_{p}(r)=0$.

(ii) For any positive number (including nonintegers) $\beta$ $\neq 1,2, \ldots, p$, by using the relation [20]

$$
\int_{-1}^{1} f(\widetilde{r}) P_{p}(\widetilde{r}) d \widetilde{r}=\frac{1}{2^{p} p !} \int_{-1}^{1} \frac{d^{p} f(\widetilde{r})}{d \widetilde{r}^{p}}\left(1-\widetilde{r}^{2}\right)^{p} d \widetilde{r},
$$

it is straightforward to obtain

$$
\int_{-1}^{1} d \widetilde{r}(1-\widetilde{r})^{\beta-1} P_{p}(\widetilde{r})=\frac{(-1)^{p} 2^{\beta}[\Gamma(\beta)]^{2}}{\Gamma(\beta-p) \Gamma(\beta+p+1)}
$$

with $\Gamma$ denoting the Gamma function [20]. Consequently, we have

$$
\begin{aligned}
\int_{0}^{l} d r r^{2 \beta} K_{p}(r)= & l^{2 \beta+2}\left[\frac{(p+1)}{2^{\beta+2}(2 \beta+1)}\right] \\
& \times \int_{-1}^{1} d \widetilde{r}(1-\widetilde{r})^{\beta-1}\left[P_{p}(\widetilde{r})-P_{p+1}(\widetilde{r})\right] \\
= & l^{2 \beta+2}\left[\frac{(-1)^{p}(p+1) \Gamma(\beta) \Gamma(\beta+1)}{(4 \beta+2) \Gamma(\beta-p) \Gamma(\beta+p+2)}\right] .
\end{aligned}
$$


[1] A. Pimpinelli and J. Villain, Physics of Crystal Growth (Cambridge University Press, Cambridge, U.K., 1998).

[2] A. L. Barabási and H. E. Stanley, Fractal Concepts in Surface Growth (Cambridge University Press, New York, 1995).

[3] J. Krug, Adv. Phys. 46, 139 (1997).

[4] T. Halpin-Healy and Y.-C. Zhang, Phys. Rep. 254, 215 (1995).

[5] S. Das Sarma, C. J. Lanczycki, R. Kotlyar, and S. V. Ghaisas, Phys. Rev. E 53, 359 (1996).

[6] N.-N. Pang and W.-J. Tzeng, Int. J. Mod. Phys. B 26, 3429 (2001).

[7] H.-N. Yang, Y.-P. Zhao, G.-C. Wang, and T.-M. Lu, Phys. Rev. Lett. 76, 3774 (1996).

[8] J. H. Jeffries, J. K. Zuo, and M. M. Craig, Phys. Rev. Lett. 76, 4931 (1996).

[9] M. Dubé et al., Eur. Phys. J. B 15, 701 (2000).

[10] S. Morel, J. Schmittbuhl, J. M. López, and G. Valentin, Phys. Rev. E 58, 6999 (1998).

[11] S. Huo and W. Schwarzacher, Phys. Rev. Lett. 86, 256 (2001).

[12] M. Saitou, Phys. Rev. B 66, 073416 (2002).
[13] A. Brú et al., Phys. Rev. Lett. 81, 4008 (1998).

[14] J. J. Ramasco, J. M. López, and M. A. Rodríguez, Phys. Rev. Lett. 84, 2199 (2000), and references therein.

[15] N.-N. Pang and W.-J. Tzeng, Phys. Rev. E 61, 3212 (2000).

[16] N.-N. Pang and W.-J. Tzeng, Phys. Rev. E 61, 3559 (2000).

[17] F. Family and T. Vicsek, J. Phys. A 18, L75 (1985).

[18] K. Hu et al., Phys. Rev. E 64, 011114 (2001), and references therein.

[19] N.-N. Pang and W.-J. Tzeng, Phys. Rev. E 69, 031108 (2004).

[20] See, for example, An Atlas of Functions, edited by J. Spanier and K. B. Oldham (Springer-Verlag, Berlin, 1987); Integrals and Series, edited by A. P. Prudnikov, Y. A. Brychkov, and O. I. Marichev (Gordon and Breach, New York, 1986).

[21] A. Chame and F. D. A. Aarão Reis, e-print cond-mat/0403033.

[22] J. Buceta, J. Pastor, M. A. Rubio, and F. J. de la Rubia, Phys. Rev. E 61, 6015 (2000).

[23] M. Siegert, Phys. Rev. E 53, 3209 (1996).

[24] J. Krug, Phys. Rev. Lett. 72, 2907 (1994). 